\title{
Labyrinthe
}

17 | 2004 (1)

Jacques Rancière, l'indiscipliné

\section{Pensées fantômes}

\section{Laurent Dubreuil}

\section{OpenEdition}

\section{Journals}

Édition électronique

URL : http://journals.openedition.org/labyrinthe/177

DOI : $10.4000 /$ labyrinthe. 177

ISSN : 1950-6031

Éditeur

Hermann

Édition imprimée

Date de publication : 15 avril 2004

Pagination : 83-86

Référence électronique

Laurent Dubreuil, «Pensées fantômes », Labyrinthe [En ligne], 17 | 2004 (1), mis en ligne le 13 juin 2008, consulté le 02 mai 2019. URL : http://journals.openedition.org/labyrinthe/177 ; DOI : 10.4000/ labyrinthe.177

Propriété intellectuelle 


\section{PENSÉES FANTÔMES}

Laurent DUBREUIL

Rancière, Derrida. Tous deux se défient des exorcismes, surtout dans la philosophie, la politique. Au contraire, il faudrait «penser le fantôme $e^{1}$ » ou « penser avec les spectres » $(A B, 201)$. Reste à savoir quels fantômes, quels spectres ces deux-là recherchent, et s'ils se croisent vraiment.

Qu'est-ce qu'un fantôme? qu'est l'effectivité ou la présence d'un spectre, c'est-à-dire de ce qui semble rester aussi ineffectif, virtuel, inconsistant qu'un simulacre?

demande Derrida ${ }^{2}$. Pour lui, la hantise peut faire pièce à toute présence, et l' «hantologie ${ }^{3} »$ qu'il appelle n'est pas l'ontologie: «Hanter ne veut pas dire être présent ${ }^{4}$. » Le spectre se dérobe, effectif dans l'évanescence. Penser dans l'impossibilité de la hantise revient finalement à la pratique de la déconstruction ${ }^{5}$, ni ceci ni cela.

Les fantômes de Rancière, je les vois nonpareils. Chez lui, ils " témoignent de la différence de chaque un à lui-même » $(A B, 201)$, et cette inscription de l'écart pourrait rappeler Derrida. Sauf que le spectral de la déconstruction voudrait sans doute faire sauter jusqu'à l'énoncé même du chaque et d'un rapport à soi. Sauf que l'accent chez Rancière est aussi sur le témoin. Les fantômes et les spectres témoignent de ce monde en ce monde, ils ne s'en échappent pas, ils s'y maintiennent. Ils sont les «témoins ${ }^{6} »(A B, 201)$ de l'exception qu'ils sont. Chaque spectre, «c'est un phare allumé sur mille citadelles », c'est en somme « le meilleur témoignage ${ }^{7} »$. Ou encore : ces fantômes ont l'air d'Idées majuscules, tombées du ciel platonicien jusqu'à nous.

1. Jacques Derrida, Spectres de Marx, Paris, Galilée, 1993, p. 52.

2. Id., p. 31.

3. Id., p. 89.

4. Id., p. 255.

5. Id., p. 24, n. 1 .

6. Voir « la fin des "spectres", c'est la fin des témoins ».

7. Charles Baudelaire, Les Fleurs du mal, « Les Phares », dans Euvres complètes, Paris, Laffont, 1980. 
En ce point, un détour.

Sans doute [la Clairon] s'est fait un modèle auquel elle a d'abord cherché à se conformer; sans doute elle a conçu ce modèle le plus haut, le plus grand, le plus parfait qu'il lui a été possible; mais ce modèle qu'elle a emprunté de l'histoire ou que son imagination a créé comme un grand fantôme, ce n'est pas elle ${ }^{8}$.

Dans son fameux Paradoxe, Diderot invoque fantômes et spectres pour désigner le processus de différence qu'éprouve chaque acteur. Diderot répond ironiquement à la théorie qu'expose Platon dans $L a$ République. Au lieu d'une dégradation inexorable de l'Idée dans le devenir artistique, chez Diderot, le « modèle idéal ${ }^{9}$ » est le dernier stade, le fantôme de l'acteur, plus grand que tout.

Mon ami, il y a trois modèles, l'homme de la nature, l'homme du poète, l'homme de l'acteur. Celui de la nature est moins grand que celui du poète, et celui-ci moins grand encore que celui du grand comédien, le plus exagéré de tous ${ }^{10}$.

Non seulement le Ciel s'est vidé (on commence avec la nature), mais l'Idée supérieure est au bout du compte (et non plus au début). Voilà qui «nous épouvante», comme

les enfants s'épouvantent les uns les autres $[\ldots]$ en s'agitant et en imitant de leur mieux la voix rauque et lugubre d'un fantôme qu'ils contrefont... ${ }^{11}$.

Le fantôme est le modèle idéal, la plus grande perfection possible. Diderot renverse l'attaque de Platon qui voulait dévaloriser la représentation poétique en la présentant comme un jeu spectral. Les termes phantasma ou eidôlon désignent la chose la plus éloignée de l'Idée ${ }^{12}$. Cela s'est souvent dit fantômes en français, comme dans la traduction

8. Denis Diderot, Paradoxe sur le comédien, Paris, Hermann, 1996, p. 30.

9. Id., passim, par exemple p. 44, 65, 102.

10. Id., p. 103.

11. Id., p. 104.

12. Voir en particulier Platon, La République, X, 598b-599a. 
Humblot de La République ${ }^{13}$ parue quelques années avant la rédaction du Paradoxe sur le comédien : «Encore ce qu'il en prend n'est-il qu'un phantôme » (eidôlon, 598b), « ces sortes d'ouvrages, qui après tout ne sont que des fantômes » (phantasmata, 599a) - ou comme, beaucoup plus tard, dans la traduction Budé: "Cette partie n'est qu'un fantôme », « ces poètes ne créent que des fantômes » ${ }^{14}$. À contre-pied, Diderot utilise le fantôme pour trouver la valeur exceptionnelle du réel, qui ne demeure pas simple chose de l'esprit mais s'éprouve matériellement dans le jeu de l'acteur. Il tente de comprendre l'art sans la théorie de la dégradation par la mimêsis, tout en gardant le fait mimétique.

En quoi la posture de Jacques Rancière est assez proche: se débarrasser radicalement de l'imitation et, pourtant... Bien sûr, il le dit, le répète, il condamne la mimétique et connaît son Platon. Mais il y revient tellement. Il voudrait se sauver du topos horatos et sauver quelque Idée néanmoins - même méconnaissable sous la forme des spectres -, tandis que Derrida marquait l'écart entre ses fantômes et tout « simulacre », repris du simulacrum latin qui transpose habituellement l'eidôlon.

Autre rencontre impossible, à propos de Mallarmé:

Toujours le « livre de vers » apparaitt comme le vrai théâtre de l'esprit, le théâtre qui n'imite que l'Idée et dont tout autre art est la simple imitation. Mais jamais Mallarmé n' arrive à penser ce modèle premier comme l'imitation de ses imitations. En ce recours infini, Jacques Derrida loua naguère la subversion par Mallarmé du système platonicien de l'idée-modèle et de la copie. Mais deux choses sont à distinguer. Mallarmé congédie l'art de la représentation et l'idée-modèle, mais il maintient pour le poème un statut mimétique : le poème n'imite aucun modèle, mais il trace sensiblement le mouvement de l'idée, l'idée comme le mouvement de son propre jaillissement (Mall., 95).

Une référence elliptique est ici faite à «La double séance », où Derrida commente « Mimique » de Mallarmé, un texte où précisément « le Mime » est « un fantôme blanc ». Commentant le poème, Derrida lisait alors « un mouvement de simulacre (non-platonicien) », faisant que

13. Platon, La République, Paris, Humblot, 1765.

14. Id., Paris, Les Belles Lettres, 1967. 
« le paradigme clinique de Platon ne fonctionne plus ${ }^{15}$ ». Rancière rectifie, ou bien il déplace l'explication. Oui, Mallarmé rejette le modèle idéal et sa représentation inférieure. Mais il aurait pu aller plus loin, si loin que d'imitation en imitation, la mimétique même soit absolument différente d'elle-même. En d'autres termes (ceux que Mallarmé ne serait pas « arriv[é] à penser »): l'imitation qui perd la référence assignable à ce qui est «premier» est capable de ne plus (se) copier. Témoin de sa différence, elle entre dans un régime fantomatique.

Voilà une tâche de pensée, nous confierait alors Rancière. Il faut reprendre ce qui s'est dit, l'imiter de telle sorte qu'on n'ait plus de copie ni d'original. « La mimétique antimimétique de l'Idée » chez Mallarmé (Mall., 101) a valeur d'exemple. Donc il convient de la déplacer toujours, comme on doit transporter les Idées platoniciennes. Rancière veut échapper aux mimétiques pour les ressaisir par différence. Mallarmé cette fois, Mandelstam une autre fois disant que «les symbolistes n'ont ni mots ni choses, seulement des fantômes de mots et des fantômes de choses » $(C M, 41)$. Ils n'ont pas les fantômes absolument; et Mandelstam aussi, etc. Dans l'optique de Derrida, le retour des spectres dans Rancière serait un effet de revenance du spectral. Dans la logique de l'œuvre de Rancière, ces fantômes sont les témoins d'une ambition générale. Présenter une pensée qui déplace les autres et se déplace encore. Construire, dans ces glissements et ces fuites, les fantômes de sa propre pensée.

15. Jacques Derrida, La Dissémination, Paris, Seuil, 1972, rééd. 1993, p. 276. 